\title{
Radiative leptonic decays on the lattice
}

\author{
Christopher Kane $^{a}$, Christoph Lehner ${ }^{b, c}$, Stefan Meinel ${ }^{* a, d}$, Amarjit Soni ${ }^{c}$ \\ ${ }^{a}$ Department of Physics, University of Arizona, Tucson, AZ 85721, USA \\ ${ }^{b}$ Department of Physics, University of Regensburg, 93040 Regensburg, Germany \\ ${ }^{c}$ Physics Department, Brookhaven National Laboratory, Upton, NY 11973, USA \\ ${ }^{d}$ RIKEN BNL Research Center, Brookhaven National Laboratory, Upton, NY 11973, USA
}

E-mail: smeinel@email.arizona.edu

Adding a hard photon to the final state of a leptonic pseudoscalar-meson decay lifts the helicity suppression and can provide sensitivity to a larger set of operators in the weak effective Hamiltonian. Furthermore, radiative leptonic $B$ decays at high photon energy are well suited to constrain the first inverse moment of the $B$-meson light-cone distribution amplitude, an important parameter in the theory of nonleptonic $B$ decays. We demonstrate that the calculation of radiative leptonic decays is possible using Euclidean lattice QCD, and present preliminary numerical results for $D_{s}^{+} \rightarrow \ell^{+} v \gamma$ and $K^{-} \rightarrow \ell^{-} \bar{v} \gamma$.

37th International Symposium on Lattice Field Theory - Lattice2019

16-22 June 2019

Wuhan, China

${ }^{*}$ Speaker. 

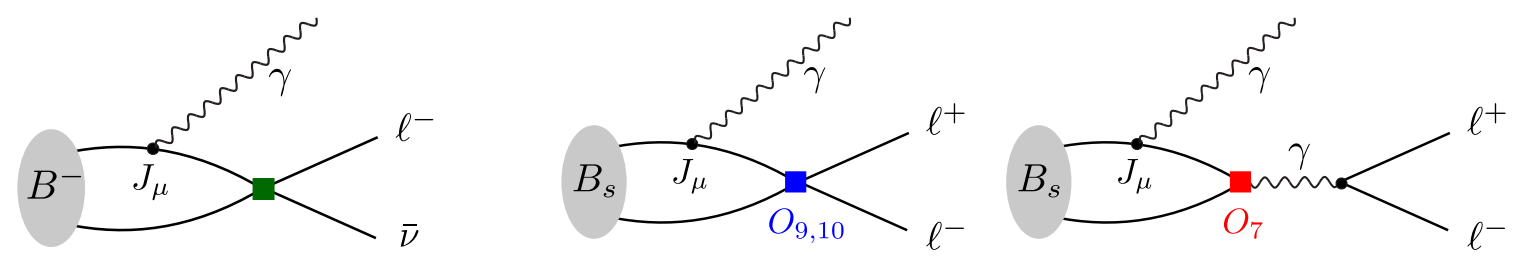

Figure 1: Left: A diagram contributing to $B^{-} \rightarrow \ell^{-} \bar{v} \gamma$, where the green square corresponds to $W$-boson exchange in the Standard Model and comes with a factor of $V_{u b}$. Right: two diagrams contributing to $B_{s} \rightarrow \ell^{+} \ell^{-} \gamma$, via the operators $O_{7,9,10}$ (defined, for example, in Ref. [1]).

\section{Introduction}

Radiative leptonic decays of pseudoscalar mesons probe both the weak interaction and the hadronic structure in useful ways. Adding a sufficiently energetic photon to the final state can actually increase the branching fraction [2], as it removes the helicity suppression. Perhaps the most interesting example is $B^{-} \rightarrow \ell^{-} \bar{v} \gamma$, shown in Fig. 1 (left). For large $E_{\gamma}^{(0)}$, this process is the cleanest probe of the first inverse moment of the $B$-meson light-cone distribution amplitude, $1 / \lambda_{B}=$ $\int_{0}^{\infty} \frac{\Phi_{B^{+}}(\omega)}{\omega} d \omega$, an important input in QCD-factorization predictions for nonleptonic $B$ decays that is presently poorly determined $[3,4,5,6,7,8,9]$. A recent search for this decay by Belle gave an upper limit $\mathscr{B}\left(B^{-} \rightarrow \ell^{-} \bar{v} \gamma, E_{\gamma}^{(0)}>1 \mathrm{GeV}\right)<3.0 \times 10^{-6}$, close to the Standard-Model expectation [10]. Lattice QCD results for the $B^{-} \rightarrow \ell^{-} \bar{v} \gamma$ form factors could be used to constrain $\lambda_{B}$. Also very interesting are the flavor-changing neutral-current decays $B^{0} \rightarrow \ell^{+} \ell^{-} \gamma$ and $B_{s} \rightarrow \ell^{+} \ell^{-} \gamma$ (shown in Fig. 1, right). While the purely leptonic decays are sensitive to $C_{10, S, P}-C_{10, S, P}^{\prime}$ only, the radiative leptonic decays probe all Wilson coefficients in the weak effective Hamiltonian, including $C_{9}$, in which global fits of experimental results for other $b \rightarrow s \ell^{+} \ell^{-}$decays indicate a deviation from the Standard Model that violates lepton flavor universality (LFU) [1]. Since the radiative leptonic decays are not helicity-suppressed, they are well-suited for testing LFU with light leptons [11, 12]. For the charmed-meson radiative leptonic decays $D^{+} \rightarrow e^{+} v \gamma$ and $D_{s}^{+} \rightarrow e^{+} v \gamma$, the BESIII collaboration has reported upper limits on the branching fractions with $E_{\gamma}^{(0)}>10 \mathrm{MeV}$ of $3.0 \times 10^{-5}$ and $1.3 \times 10^{-4}$, respectively [13,14]. Finally, in contrast to the heavy-meson decays, there are already precise measurements of the differential branching fractions of $K^{-} \rightarrow e^{-} \bar{v} \gamma, K^{-} \rightarrow \mu^{-} \bar{v} \gamma$, $\pi^{-} \rightarrow e^{-} \bar{v} \gamma$, and $\pi^{-} \rightarrow \mu^{-} \bar{v} \gamma$, as reviewed in Ref. [15]. These decay modes can therefore be used to test the lattice QCD methods.

In the following, we show how radiative leptonic decays can be calculated on a Euclidean lattice, and we present early numerical results. One of us previously reported on this project at the Lattice 2018 conference [16]. At Lattice 2019, radiative leptonic decays were also discussed by G. Martinelli [17].

\section{Hadronic tensor and form factors}

To define the form factors for charged-current radiative leptonic decays of pseudoscalar mesons, we use the notation for $B^{-} \rightarrow \ell^{-} \bar{v} \gamma$. The quark electromagnetic and weak currents are given by $J_{\mu}=\sum_{q} e_{q} \bar{q} \gamma_{\mu} q$ and $J_{v}^{\text {weak }}=\bar{u} \gamma_{v}\left(1-\gamma_{5}\right) b$. The decay amplitude depends on the hadronic tensor, 
which is defined as

$$
T_{\mu \nu}=-i \int d^{4} x e^{i p_{\gamma} \cdot x}\left\langle 0\left|\mathrm{~T}\left(J_{\mu}(x) J_{v}^{\mathrm{weak}}(0)\right)\right| B^{-}\left(\mathbf{p}_{B}\right)\right\rangle
$$

in Minkowski space. Throughout this work, we asssume that the photon is real, i.e., $p_{\gamma}^{2}=0$. The hadronic tensor can be decomposed as [7]

$$
T_{\mu v}=\varepsilon_{\mu \nu \tau \rho} p_{\gamma}^{\tau} v^{\rho} F_{V}+i\left[-g_{\mu v}\left(p_{\gamma} \cdot v\right)+v_{\mu}\left(p_{\gamma}\right)_{v}\right] F_{A}-i \frac{{ }^{v_{\mu} v_{v}}}{p_{\gamma} \cdot v} m_{B} f_{B}+\left(p_{\gamma}\right)_{\mu} \text {-terms, }
$$

where $p_{B}=m_{B} v$ and the $\left(p_{\gamma}\right)_{\mu}$-terms will disappear when contracting with the photon polarization vector. The form factors $F_{V}$ and $F_{A}$ are functions of the photon energy in the $B$-meson rest frame, $E_{\gamma}^{(0)}=p_{\gamma} \cdot V=\left(m_{B}^{2}-q^{2}\right) /\left(2 m_{B}\right)$. Also appearing in Eq. (2.2) is the $B$-meson decay constant $f_{B}$.

To prepare for the discussion in the next section, it is useful to write down the spectral representation of $T_{\mu \nu}$ in Minkowski space for the two different time orderings of the currents. By inserting complete sets of energy/momentum eigenstates and performing the time integrals, we find

$$
\begin{aligned}
T_{\mu \nu}^{<} & =-i \int_{-\infty(1-i \varepsilon)}^{0} d t \quad e^{i E_{\gamma} t} \int d^{3} x e^{-i \mathbf{p}_{\gamma} \cdot \mathbf{x}}\left\langle 0\left|J_{v}^{\mathrm{weak}}(0) J_{\mu}(t, \mathbf{x})\right| B^{-}\left(\mathbf{p}_{B}\right)\right\rangle \\
& =-\sum_{n} \frac{1}{2 E_{n,\left(\mathbf{p}_{B}-\mathbf{p}_{\gamma}\right)}} \frac{\left\langle 0\left|J_{v}^{\mathrm{weak}}(0)\right| n\left(\mathbf{p}_{B}-\mathbf{p}_{\gamma}\right)\right\rangle\left\langle n\left(\mathbf{p}_{B}-\mathbf{p}_{\gamma}\right)\left|J_{\mu}(0)\right| B\left(\mathbf{p}_{B}\right)\right\rangle}{E_{\gamma}+E_{n,\left(\mathbf{p}_{B}-\mathbf{p}_{\gamma}\right)}-E_{B}-i \varepsilon}, \\
T_{\mu \nu}^{>} & =-i \int_{0}^{\infty(1-i \varepsilon)} d t \quad e^{i E_{\gamma} t} \int d^{3} x e^{-i \mathbf{p}_{\gamma} \cdot \mathbf{x}}\left\langle 0\left|J_{\mu}(t, \mathbf{x}) J_{v}^{\text {weak }}(0)\right| B^{-}\left(\mathbf{p}_{B}\right)\right\rangle \\
& =\sum_{m} \frac{1}{2 E_{m, \mathbf{p}_{\gamma}}} \frac{\left\langle 0\left|J_{\mu}(0)\right| m\left(\mathbf{p}_{\gamma}\right)\right\rangle\left\langle m\left(\mathbf{p}_{\gamma}\right)\left|J_{v}^{\text {weak }}(0)\right| B\left(\mathbf{p}_{B}\right)\right\rangle}{E_{\gamma}-E_{m, \mathbf{p}_{\gamma}}-i \varepsilon}
\end{aligned}
$$

(in infinite volume, the sums over $n$ and $m$ include integrals over the continuous spectrum of multiparticle states).

\section{Extracting the hadronic tensor from a Euclidean three-point function}

In this section, we show that $T_{\mu \nu}$ can be extracted from the Euclidean three-point function

$$
C_{\mu v}\left(t, t_{B}\right)=\int d^{3} x \int d^{3} y e^{-i \mathbf{p}_{\gamma} \cdot \mathbf{x}} e^{i \mathbf{p}_{B} \cdot \mathbf{y}}\left\langle J_{\mu}(t, \mathbf{x}) J_{v}^{\text {weak }}(0, \mathbf{0}) \phi_{B}^{\dagger}\left(t_{B}, \mathbf{y}\right)\right\rangle,
$$

where $\phi_{B} \sim \bar{u} \gamma_{5} b$ is an interpolating field for the $B$ meson, and $t, t_{B}$ now denote the Euclidean time. We define the integrals

$$
I_{\mu v}^{<}\left(t_{B}, T\right)=\int_{-T}^{0} d t e^{E_{\gamma} t} C_{\mu v}\left(t, t_{B}\right), \quad I_{\mu v}^{>}\left(t_{B}, T\right)=\int_{0}^{T} d t e^{E_{\gamma} t} C_{\mu v}\left(t, t_{B}\right),
$$

with a finite integration range $T$. Here we take $t_{B}$ to be large and negative (with $t_{B}<-T$ ), such that ground-state saturation is achieved for the $B$ meson. Inserting again complete sets of energy/momentum eigenstates, we find, for the first time ordering,

$$
\begin{aligned}
I_{\mu \nu}^{<}\left(t_{B}, T\right)= & \left\langle B\left(\mathbf{p}_{B}\right)\left|\phi_{B}^{\dagger}(0)\right| 0\right\rangle \frac{1}{2 E_{B}} e^{E_{B} t_{B}} \\
& \times \sum_{n} \frac{1}{2 E_{n,\left(\mathbf{p}_{B}-\mathbf{p}_{\gamma}\right)}} \frac{\left\langle 0\left|J_{v}^{\mathrm{weak}}(0)\right| n\left(\mathbf{p}_{B}-\mathbf{p}_{\gamma}\right)\right\rangle\left\langle n\left(\mathbf{p}_{B}-\mathbf{p}_{\gamma}\right)\left|J_{\mu}(0)\right| B\left(\mathbf{p}_{B}\right)\right\rangle}{E_{\gamma}+E_{n,\left(\mathbf{p}_{B}-\mathbf{p}_{\gamma}\right)}-E_{B}} \\
& \times\left(1-e^{-\left(E_{\gamma}+E_{n,\left(\mathbf{p}_{B}-\mathbf{p}_{\gamma}\right)}-E_{B}\right) T}\right) .
\end{aligned}
$$


The sum over states in Eq. (3.3) differs from the sum in Eq. (2.3) by the factor in the last line. However, the exponential $e^{-\left(E_{\gamma}+E_{n,\left(\mathbf{p}_{B}-\mathbf{p} \gamma\right)}-E_{B}\right) T}$ will vanish for large $T$ if $E_{\gamma}+E_{n,\left(\mathbf{p}_{B}-\mathbf{p}_{\gamma}\right)}>E_{B}$. Because the states $\left|n\left(\mathbf{p}_{B}-\mathbf{p}_{\gamma}\right)\right\rangle$ have the same quark-flavor quantum numbers as the $B$ meson, we have $E_{n,\left(\mathbf{p}_{B}-\mathbf{p}_{\gamma}\right)} \geq E_{B,\left(\mathbf{p}_{B}-\mathbf{p}_{\gamma}\right)}=\sqrt{m_{B}^{2}+\left(\mathbf{p}_{B}-\mathbf{p}_{\gamma}\right)^{2}}$. Thus, we need $\sqrt{\mathbf{p}_{\gamma}^{2}}+\sqrt{m_{B}^{2}+\left(\mathbf{p}_{B}-\mathbf{p}_{\gamma}\right)^{2}}>$ $\sqrt{m_{B}^{2}+\mathbf{p}_{B}^{2}}$. This is in fact always true if $\mathbf{p}_{\gamma} \neq 0$.

For the other time ordering, we find

$$
\begin{aligned}
I_{\mu \nu}^{>}\left(t_{B}, T\right)= & -\left\langle B\left(\mathbf{p}_{B}\right)\left|\phi_{B}^{\dagger}(0)\right| 0\right\rangle \frac{1}{2 E_{B}} e^{E_{B} t_{B}} \\
& \times \sum_{m} \frac{1}{2 E_{m, \mathbf{p}_{\gamma}}} \frac{\left\langle 0\left|J_{\mu}(0)\right| m\left(\mathbf{p}_{\gamma}\right)\right\rangle\left\langle m\left(\mathbf{p}_{\gamma}\right)\left|J_{v}^{\mathrm{weak}}(0)\right| B\left(\mathbf{p}_{B}\right)\right\rangle}{E_{\gamma}-E_{m, \mathbf{p}_{\gamma}}}\left(1-e^{\left(E_{\gamma}-E_{m, \mathbf{p} \gamma}\right) T}\right) .
\end{aligned}
$$

The unwanted exponential $e^{\left(E_{\gamma}-E_{m, \mathbf{p} \gamma}\right) T}$ in the last line goes to zero for large $T$ if $E_{m, \mathbf{p}_{\gamma}}>E_{\gamma}$. Because the states $\left|m\left(\mathbf{p}_{\gamma}\right)\right\rangle$ are hadronic and have nonzero masses, their energies are larger than the energy of a photon with the same spatial momentum, showing that this condition is also always satisfied. In summary, for $\mathbf{p}_{\gamma} \neq 0$,

$$
T_{\mu \nu}=-\lim _{T \rightarrow \infty} \lim _{t_{B} \rightarrow-\infty} \frac{2 E_{B} e^{-E_{B} t_{B}}}{\left\langle B\left(\mathbf{p}_{B}\right)\left|\phi_{B}^{\dagger}(0)\right| 0\right\rangle} I_{\mu v}\left(t_{B}, T\right),
$$

where $I_{\mu v}$ is the integral from $-T$ to $T$. The energy $E_{B}$ and the overlap factor $\left\langle B\left(\mathbf{p}_{B}\right)\left|\phi_{B}^{\dagger}(0)\right| 0\right\rangle$ can be obtained from the two-point function $\int d^{3} x e^{-i \mathbf{p}_{B} \cdot \mathbf{x}}\left\langle\phi_{B}(t, \mathbf{x}) \phi_{B}^{\dagger}(0)\right\rangle$.

Note that similar nonlocal matrix elements appear in processes with two photons, whose lattice calculation has been discussed, for example, in Refs. [18, 19, 20].

\section{Preliminary numerical results}

In this section, we present some early numerical results for the $D_{s}^{+} \rightarrow \ell^{+} v \gamma$ and $K^{-} \rightarrow \ell^{-} \bar{v} \gamma$ form factors. These results are from only 25 configurations of the " $24 \mathrm{I}$ " RBC/UKQCD ensemble

$\mu=1, \nu=2(\mathrm{~V}), \operatorname{Re}$

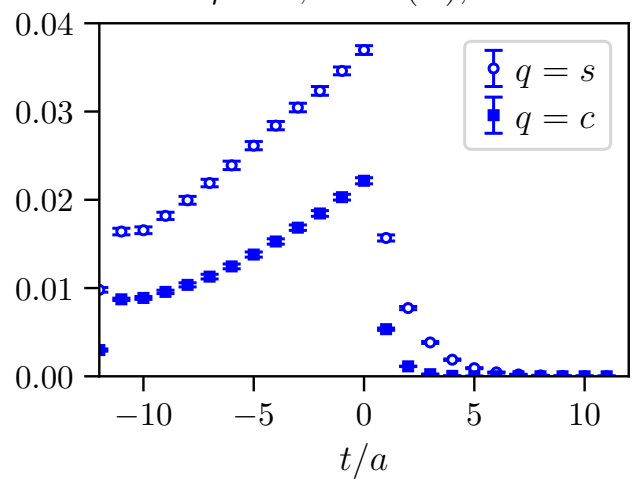

$\mu=1, \nu=1(\mathrm{~A}), \operatorname{Im}$

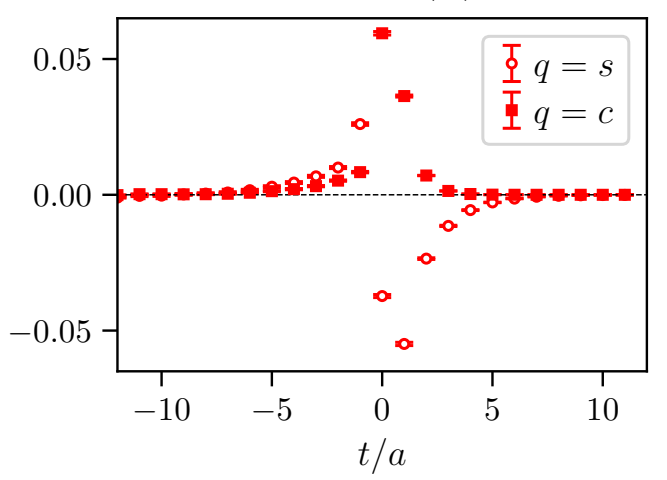

Figure 2: The unintegrated, scaled three-point functions $-\frac{2 E_{D_{s}} e^{-E_{D_{s}} t_{s}}}{\left\langle D_{s}\left(\mathbf{p}_{D_{s}}\right) \phi_{D_{s}}^{\dagger}(0) \mid 0\right\rangle} C_{\mu v}\left(t, t_{D_{s}}\right)$ as a function of the electromagnetic-current insertion time $t$, for $t_{D_{s}} / a=-12$ and $\mathbf{p}_{\gamma}=(0,0,1) \frac{2 \pi}{L}$. The left plot shows a combination of indices sensitive to $F_{V}$, while the right plot shows a combination sensitive to $F_{A}$. The contributions from the $s$ and $c$ quark in the electromagnetic current are shown separately, without charge factors. 

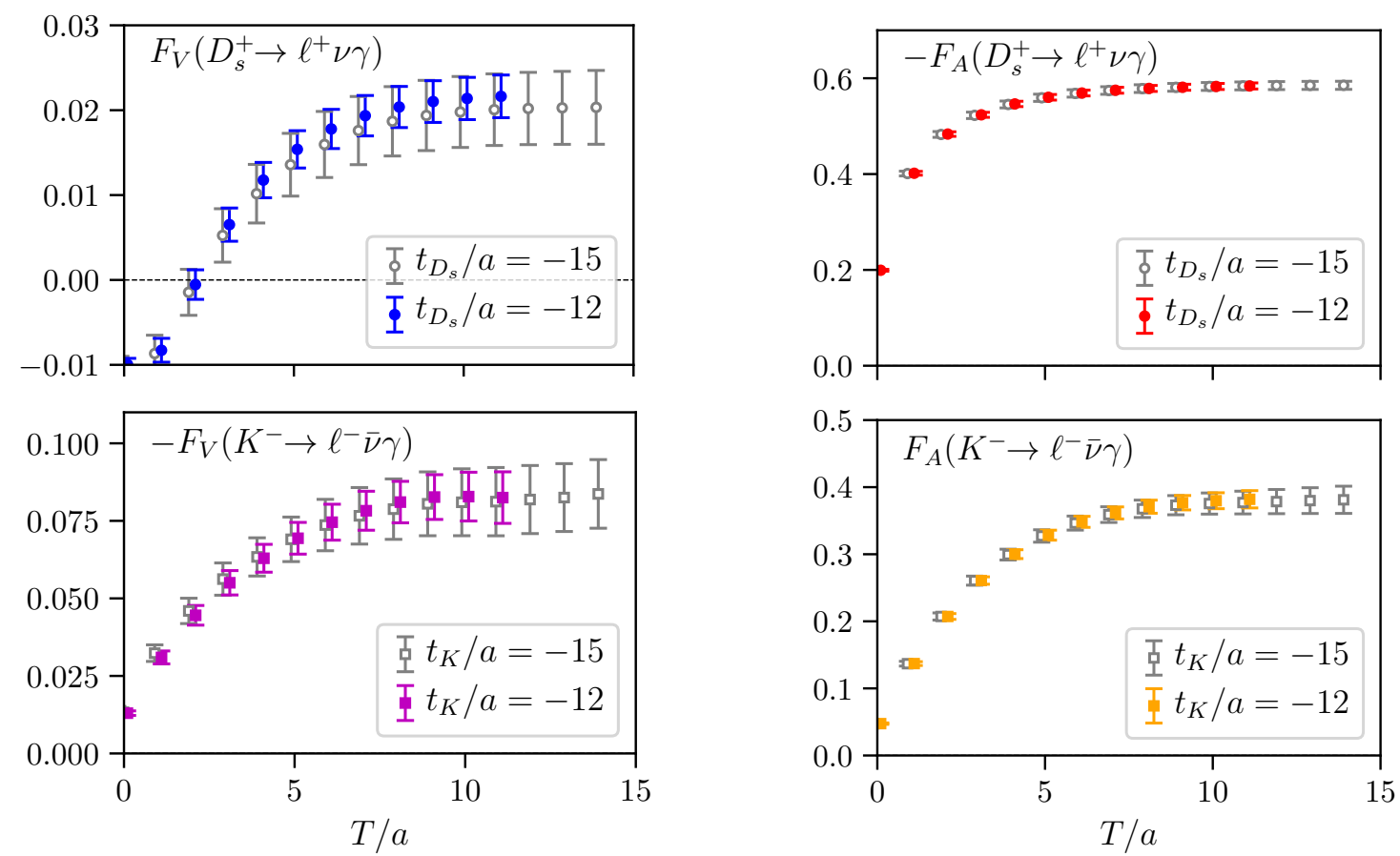

Figure 3: The $D_{s}^{+} \rightarrow \ell^{+} v \gamma$ and $K^{-} \rightarrow \ell^{-} \bar{v} \gamma$ form factors at $\mathbf{p}_{\gamma}=(0,0,1) \frac{2 \pi}{L}$ as a function of the summation range $T$, for two different meson-field insertion times.
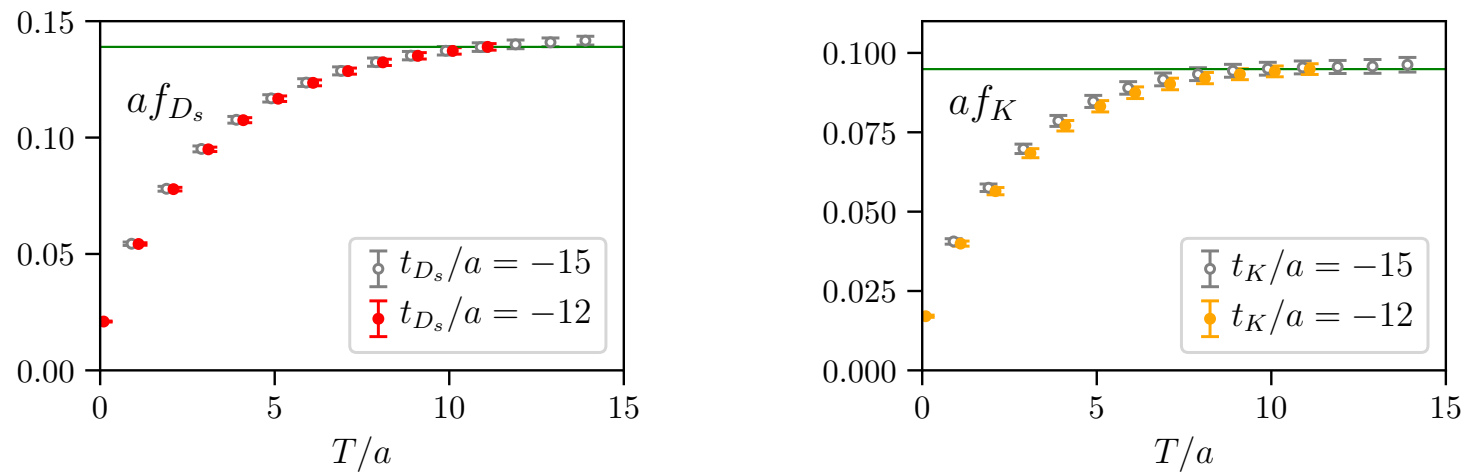

Figure 4: The $D_{s}$ and $K$ decay constants extracted from $T_{\mu v}$ at $\mathbf{p}_{\gamma}=(0,0,1) \frac{2 \pi}{L}$, as a function of the summation range $T$, for two different meson-field insertion times. For the $D_{s}$, the horizontal line shows the physical value from Ref. [21]. For the $K$, the horizontal line shows the value computed on the same ensemble with the standard method in Ref. [22].

[22] with $2+1$ flavors of domain-wall fermions and the Iwasaki gauge action, with $a^{-1}=1.785(5)$ $\mathrm{GeV}$ and $m_{\pi}=340(1) \mathrm{MeV}$. For the light and strange valence quarks, we use the same domainwall action as in Ref. [22]. The valence charm quark is implemented with a Möbius domain-wall action with stout-smeared gauge links $(N=3, \rho=0.1), L_{5} / a=12, a M_{5}=1.0, a m_{f}=0.6$ [23], which approximately corresponds to the physical charm-quark mass. We use local currents with "mostly nonperturbative" renormalization. Gaussian smearing is performed for the lighter quark in the meson interpolating field. We start with a $\mathbb{Z}_{2}$ random-wall source at the time slice of the weak current (denoted as time " 0 " here) and perform sequential inversions through the meson in- 

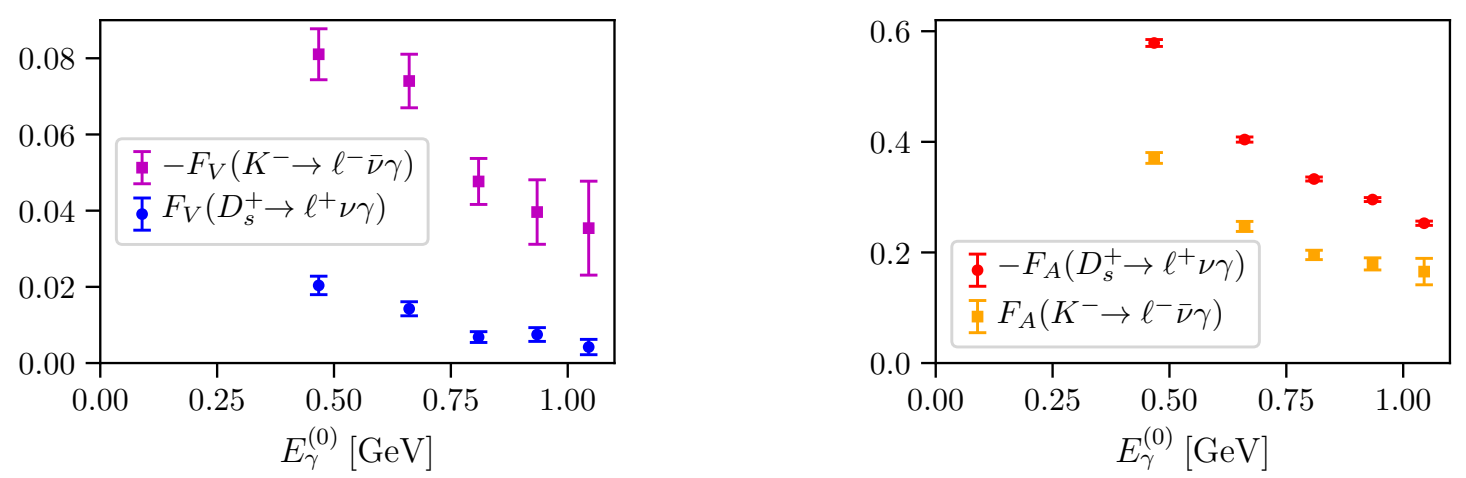

Figure 5: The $D_{s}^{+} \rightarrow \ell^{+} v \gamma$ and $K^{-} \rightarrow \ell^{-} \bar{v} \gamma$ form factors as a function of the photon energy. The results shown here were obtained with $T / a=8$ and $t_{K / D_{s}} / a=-12$. Only the statistical uncertainties are given.

terpolating field; disconnected diagrams are presently neglected. All-mode averaging [24] with 16 sloppy and 1 exact samples per configuration is employed; the 16 sloppy samples correspond to 16 different starting time slices. Our initial calculations used $\mathbf{p}_{K / D_{s}}=0$ and $\mathbf{p}_{\gamma}^{2} \in\{1,2,3,4,5\}\left(\frac{2 \pi}{L}\right)^{2}$.

Figure 2 shows examples of the $D_{s}^{+} \rightarrow \ell^{+} v \gamma$ three-point functions. Multiplying by $e^{E_{\gamma} t}$ and summing over $t$ gives $T_{\mu \nu}$ for sufficiently large summation range $T$. The form factors $F_{V}$ and $F_{A}$ extracted from $T_{\mu \nu}$ (at the lowest photon momentum) are shown as a function of $T$ in Fig. 3. The results plateau at approximately $T / a=8$. We also extracted the meson decay constants from the $v^{\mu} v^{v}$ term in $T_{\mu \nu}$. As can be seen in Fig. 4, the results agree with the known values, which is a valuable test of our calculation. Finally, Fig. 5 shows the form factors $F_{V}$ and $F_{A}$ as a function of the photon energy. Note that, with our current choice of momenta, all of the photon energies are above the physical region for $K^{-} \rightarrow \ell^{-} \bar{v} \gamma$. The results for $F_{A}$ are dominated by the point-like contribution equal to $-e_{\ell} f_{K / D_{s}} / E_{\gamma}^{(0)}$.

\section{Conclusions and Outlook}

We have shown that the form factors describing radiative leptonic decays can be calculated on the lattice; even though they involve a nonlocal matrix element, the use of imaginary time poses no difficulty in this case. The early results shown here for $D_{s}^{+} \rightarrow \ell^{+} v \gamma$ and $K^{-} \rightarrow \ell^{-} \bar{v} \gamma$ cover photon energies from approximately 0.5 to $1 \mathrm{GeV}$. For $K^{-} \rightarrow \ell^{-} \bar{v} \gamma$ we need to reach lower photon energies to compare with experiment; this can be achieved by using moving frames (i.e., nonzero $\mathbf{p}_{K}$ ) and/or a larger volume. To study the $B_{(s)}$ radiative leptonic decays with the domainwall action for the heavy quark, we will need to extrapolate in the mass. We are also considering calculations directly at the physical $b$-quark mass using the "relativistic heavy-quark action" [25], but, because this action is only on-shell improved, additional steps are likely needed to remove unphysical behavior occurring when the electromagnetic and weak currents get close to each other.

Acknowledgments: We thank the RBC and UKQCD Collaborations for providing the gauge-field configurations. C.K. and S.M. are supported by the US DOE, Office of Science, Office of HEP under award number DE-SC0009913. S.M. is also supported by the RIKEN BNL Research Center. A.S. and C.L. are supported in part by US DOE Contract No. DESC0012704(BNL). During a part of this work, C.L. was also supported by a DOE Office of Science Early Career Award. This work used resources at TACC that are part of XSEDE, supported by NSF grant number ACI-1548562. 
[1] J. Aebischer, W. Altmannshofer, D. Guadagnoli, M. Reboud, P. Stangl, and D. M. Straub, “B-decay discrepancies after Moriond 2019," arXiv:1903.10434.

[2] D. Atwood, G. Eilam, and A. Soni, "Pure leptonic radiative decays $B^{ \pm}, D_{s} \rightarrow \ell v \gamma$ and the annihilation graph,” Mod. Phys. Lett. A11 (1996) 1061, arXiv: hep-ph/9411367.

[3] G. P. Korchemsky, D. Pirjol, and T.-M. Yan, "Radiative leptonic decays of B mesons in QCD," Phys. Rev. D61 (2000) 114510, arXiv: hep-ph/9911427.

[4] S. Descotes-Genon and C. T. Sachrajda, "Factorization, the light cone distribution amplitude of the B meson and the radiative decay $B \rightarrow \gamma \ell v_{\ell}, "$ Nucl. Phys. B650 (2003) 356, arXiv: hep-ph/0209216.

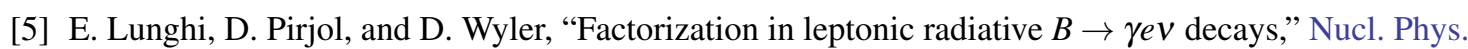
B649 (2003) 349, arXiv: hep-ph/ 0210091.

[6] V. M. Braun and A. Khodjamirian, "Soft contribution to $B \rightarrow \gamma \ell v_{\ell}$ and the $B$-meson distribution amplitude," Phys. Lett. B718 (2013) 1014, arXiv: 1210.4453.

[7] M. Beneke, V. M. Braun, Y. Ji, and Y.-B. Wei, "Radiative leptonic decay $B \rightarrow \gamma \ell v_{\ell}$ with subleading power corrections," JHEP 07 (2018) 154, arXiv: 1804.04962.

[8] Y.-M. Wang and Y.-L. Shen, "Subleading-power corrections to the radiative leptonic $B \rightarrow \gamma \ell v$ decay in QCD,” JHEP 05 (2018) 184, arXiv: 1803.06667.

[9] M. Beneke, G. Buchalla, M. Neubert, and C. T. Sachrajda, "QCD factorization for $B \rightarrow \pi \pi$ decays: Strong phases and CP violation in the heavy quark limit," Phys. Rev. Lett. 83 (1999) 1914, arXiv:hep-ph/9905312.

[10] Belle Collaboration, M. Gelb et al., "Search for the rare decay of $B^{+} \rightarrow \ell^{+} v_{\ell} \gamma$ with improved hadronic tagging,” Phys. Rev. D98 (2018) 112016, arXiv:1810.12976.

[11] D. Guadagnoli, M. Reboud, and R. Zwicky, " $B_{s}^{0} \rightarrow \ell^{+} \ell^{-} \gamma$ as a test of lepton flavor universality," JHEP 11 (2017) 184, arXiv: 1708.02649.

[12] F. Dettori, D. Guadagnoli, and M. Reboud, " $B_{s}^{0} \rightarrow \mu^{+} \mu^{-} \gamma$ from $B_{s}^{0} \rightarrow \mu^{+} \mu^{-}$," Phys. Lett. B768 (2017) 163, arXiv:1610.00629.

[13] BESIII Collaboration, M. Ablikim et al., "Search for the radiative leptonic decay $D^{+} \rightarrow \gamma e^{+} v_{e}$," Phys. Rev. D95 (2017) 071102, arXiv:1702.05837.

[14] BESIII Collaboration, M. Ablikim et al., "Search for the decay $D_{s}^{+} \rightarrow \gamma e^{+} v_{e}$," Phys. Rev. D99 (2019) 072002, arXiv:1902.03351.

[15] Particle Data Group Collaboration, M. Tanabashi et al., "Review of Particle Physics," Phys. Rev. D98 (2018) 030001.

[16] A. Soni, "Flavor anomalies \& the lattice," 2018. Presentation at Lattice 2018, East Lansing, MI, USA.

[17] RM123 Collaboration, G. Martinelli, "Electromagnetic Corrections to Decay Amplitudes: Real Emissions in Leptonic Decays," 2019. Presentation at Lattice 2019, Wuhan, China.

[18] X.-d. Ji and C.-w. Jung, "Studying hadronic structure of the photon in lattice QCD," Phys. Rev. Lett. 86 (2001) 208, arXiv: hep-lat/0101014.

[19] J. J. Dudek and R. G. Edwards, “Two Photon Decays of Charmonia from Lattice QCD,” Phys. Rev. Lett. 97 (2006) 172001, arXiv: hep-ph/ 0607140.

[20] X. Feng, S. Aoki, H. Fukaya, S. Hashimoto, T. Kaneko, J.-i. Noaki, and E. Shintani, "Two-photon decay of the neutral pion in lattice QCD," Phys. Rev. Lett. 109 (2012) 182001, arXiv: 1206.1375.

[21] Flavour Lattice Averaging Group Collaboration, S. Aoki et al., "FLAG Review 2019," arXiv:1902.08191.

[22] RBC/UKQCD Collaboration, Y. Aoki et al., "Continuum Limit Physics from 2+1 Flavor Domain Wall QCD,” Phys. Rev. D83 (2011) 074508, arXiv: 1011.0892.

[23] RBC/UKQCD Collaboration, P. A. Boyle, L. Del Debbio, N. Garron, A. Jüttner, A. Soni, J. T. Tsang, and O. Witzel, "SU(3)-breaking ratios for $D_{(s)}$ and $B_{(s)}$ mesons," arXiv:1812.08791.

[24] E. Shintani, R. Arthur, T. Blum, T. Izubuchi, C. Jung, and C. Lehner, "Covariant approximation averaging,” Phys. Rev. D91 (2015) 114511, arXiv:1402.0244.

[25] N. H. Christ, M. Li, and H.-W. Lin, "Relativistic Heavy Quark Effective Action,” Phys. Rev. D76 (2007) 074505, arXiv:hep-lat/0608006. 